\title{
Towards capturing a database of respiratory exhalations from flow visualisations
}

\section{Prateek Bahl $^{1 *}$, Charitha de Silva ${ }^{1}$, C Raina MacIntyre ${ }^{2}$, Abrar Ahmad Chughtai $^{3}$, Con Doolan ${ }^{1}$}

\author{
${ }^{1}$ UNSW Sydney, School of Mechanical and Manufacturing Engineering, Sydney, Australia \\ ${ }^{2}$ UNSW Sydney, Biosecurity Program, The Kirby Institute, Sydney, Australia \\ ${ }^{3}$ UNSW Sydney, School of Population Health, Sydney, Australia \\ *p.bahl@unsw.edu.au
}

\begin{abstract}
One of the most common modes of infection transmission is through pathogen laden droplets expelled during natural human respiratory exhalations such as speaking, coughing, and sneezing. Infection control guidelines for the prevention of respiratory infection make assumptions about two key parameters: the safe distance between an infected and healthy individual and the size of large and small droplets (Bahl et al., 2020). Studies in the past have utilised flow visualisation techniques to understand the dynamics of respiratory flows but most of them provide only qualitative data on respiratory droplets and do not provide sufficient detail to estimate accurate flow velocities (Bourouiba et al., 2014; Vansciver et al., 2011; Scharfman et al., 2016). One of the reasons this remains a demanding application is the vast range of droplet sizes that are expelled at various velocities. Here, we present an experimental framework using particle tracking to understand the flow dynamics of the expelled droplets. Three different illumination techniques were used to capture high-speed frames of different exhalations (see figure 1). The high density of droplets in case of sneezing lead to overlap of droplet trajectories with volume illumination approach, which was resolved using tailored optics to illuminate only a slice of sneeze flow. Thereafter, the image processing techniques required for precise PTV were refined to examine droplet dynamics of various exhalations (see figure 2). The techniques were applied to multiple cases of respiratory exhalations to understand subject to subject variability.

The results for sneezing revealed a mean droplet velocity of $2 \mathrm{~m} / \mathrm{s}$ to $5.4 \mathrm{~m} / \mathrm{s}$ across different subjects. Additionally, less than $1 \%$ of droplets were expelled at velocities greater than $10 \mathrm{~m} / \mathrm{s}$ and almost $80 \%$ of were expelled at velocities less than $5 \mathrm{~m} / \mathrm{s}$. These values were substantially lower than the values usually assumed in studies modelling or replicating sneezes (Xie et al., 2007; Atkinson and Wein, 2008). The results
\end{abstract}

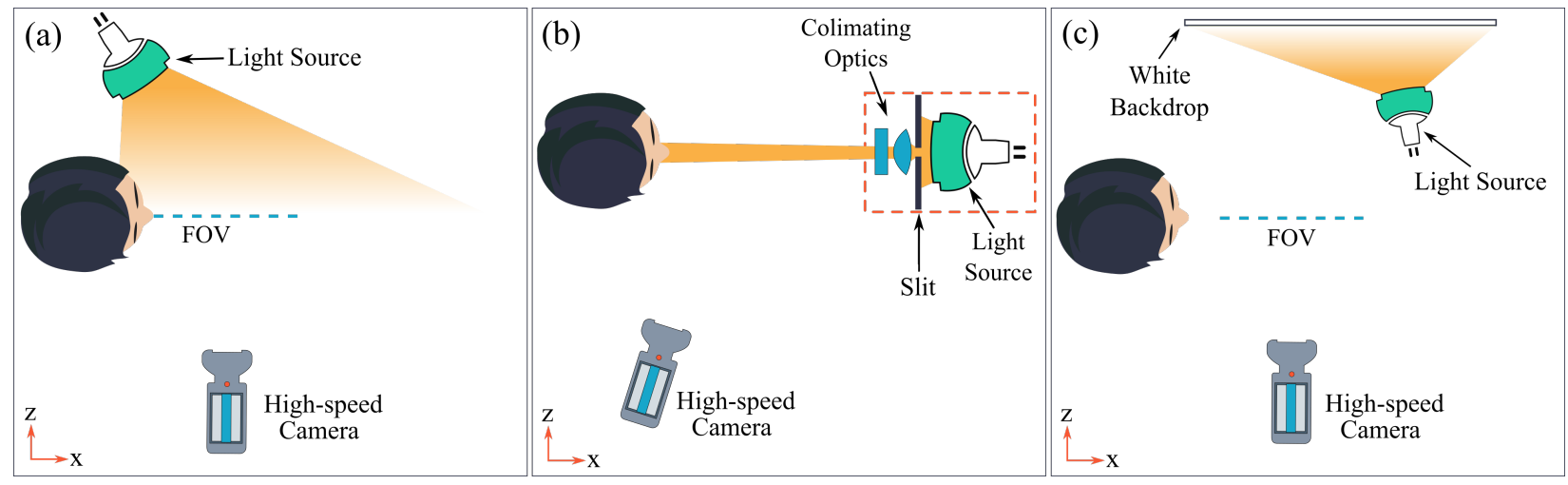

Figure 1: Schematics of setups used to capture expelled droplets using (a) volume illumination, (b) light sheet illumination, and (c) shadowgraph imaging. 

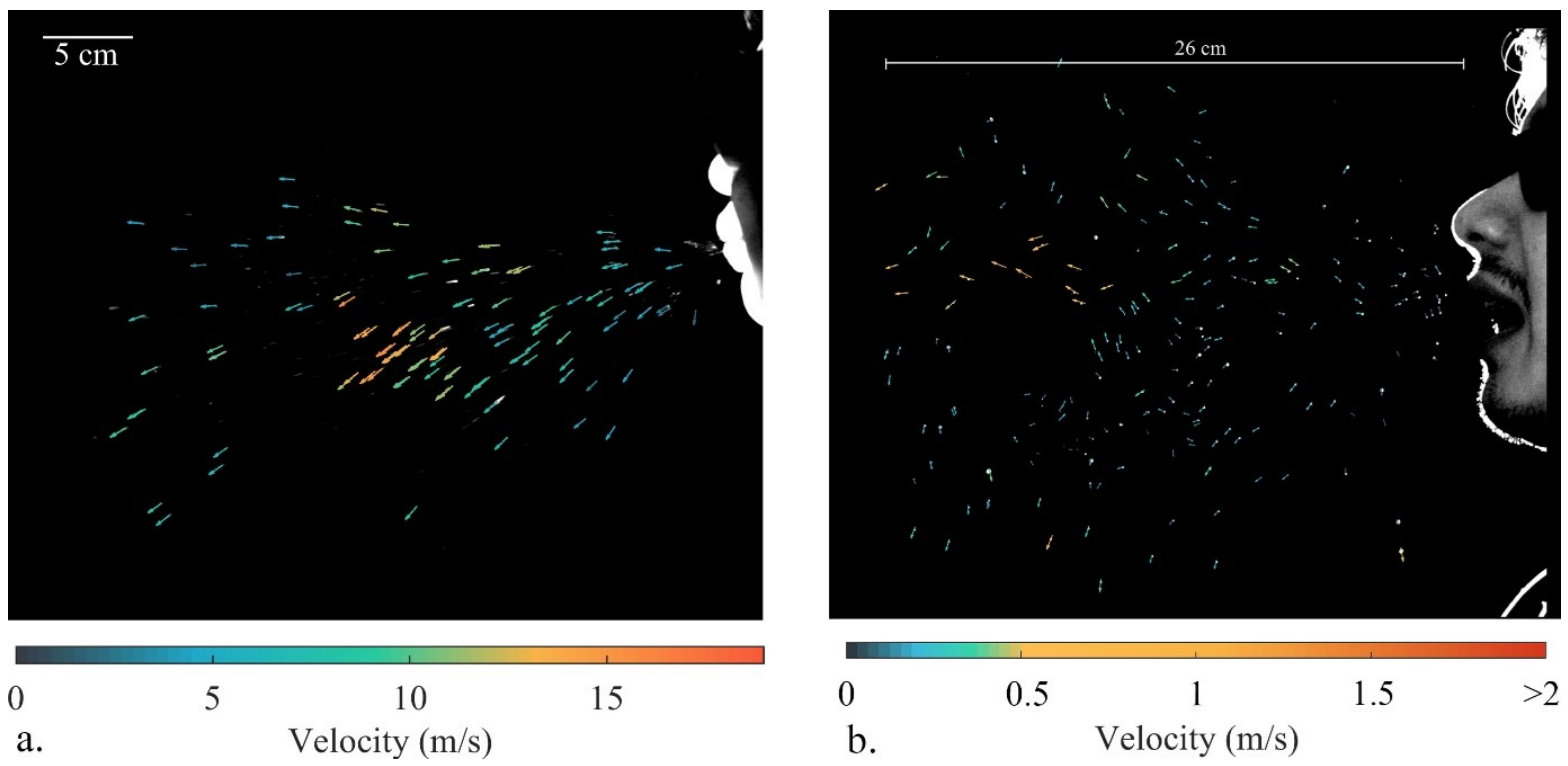

Figure 2: Velocity associated with droplets expelled during (a) Sneezing, and (b) Singing

also revealed a high variation in the droplet dynamics, even among the sneezes from the same subject. Flow direction, spread angle, and head movement were also quantified, and the results reveal substantial variation between the subjects. In the case of coughing, maximum droplet velocities observed were in the range of $10-15 \mathrm{~m} / \mathrm{s}$ however, these high velocities were detected only during the initial $0.05 \mathrm{~s}$.

This work addresses the critical gaps in the understanding of the respiratory transmission of infection by providing valuable data on the droplet dynamics of various exhalations, on which the experimental data was very limited in the existing literature. Furthermore, this data will aid in numerical modelling of respiratory flows, particularly for sneezes, as studies to date rely only on airflow data of the exhalations.

\section{References}

Atkinson MP and Wein LM (2008) Quantifying the Routes of Transmission for Pandemic Influenza. Bulletin of Mathematical Biology 70:820-867

Bahl P, Doolan C, de Silva C, Chughtai AA, Bourouiba L, and MacIntyre CR (2020) Airborne or Droplet Precautions for Health Workers Treating Coronavirus Disease 2019?. The Journal of Infectious Diseases

Bourouiba L, Dehandschoewercker E, and Bush JW (2014) Violent expiratory events: On coughing and sneezing. Journal of Fluid Mechanics 745:537-563

Scharfman B, Techet A, Bush J, and Bourouiba L (2016) Visualization of sneeze ejecta: steps of fluid fragmentation leading to respiratory droplets. Experiments in Fluids 57:24

Vansciver M, Miller S, and Hertzberg J (2011) Particle image velocimetry of human cough. Aerosol Science and Technology 45:415-422

Xie X, Li Y, Chwang AT, Ho PL, and Seto WH (2007) How far droplets can move in indoor environments revisiting the Wells evaporation-falling curve. Indoor Air 17:211-225 